\title{
MediaDASH Tool: Plataforma Web para la Codificación, Difusión y Recepción de Videos DASH
}

\author{
Miguel García-Pineda, Daniel García-Costa, Jonatan Hannecke-Esteve, \\ Santiago Felici-Castell, Jaume Segura-Garcia. \\ Departament d'Informàtica \\ Universitat de València \\ Av. de la Universitat, s/n. 46100 - Burjassot. Valencia. Spain. \\ migarpi@uv.es, daniel.garcia@uv.es, jonidhe@gmail.com, felici@uv.es, jsegura@uv.es
}

\begin{abstract}
Resumen- Hoy en día el tráfico de video en Internet significa alrededor del $65-70 \%$ del tráfico total. Se prevee que en 2019 aumente hasta el $80 \%$. Debido a esto se hace necesario optimizar y mejorar las tecnologías relacionadas con la transmisión de video para garantizar una calidad de experiencia adecuada. Es por ello que se están desarrollando nuevos protocolos de transmisión de video adaptativos basados en DASH. Debido a que su uso está aumentando en los últimos años para realizar streaming sobre HTTP. En este artículo presentamos la herramienta MediaDASH Tool, la cual es una aplicación web que permite de manera muy intuitiva poder comprimir $y$ preparar videos para su difusión con DASH. Así como su posterior visualización a través de la misma plataforma. Esta herramienta puede ser de gran utilidad para usuarios finales que quieran disponer de su sistema DASH como también para investigadores que quieran testear sus contenidos multimedia haciendo uso de estas técnicas de streaming.
\end{abstract}

Palabras Clave- DASH, MPEG-DASH, WebM-DASH, web, herramienta, codificación, streaming

\section{INTRODUCCIÓN}

Según datos del último estudio [1] realizado por Cisco Systems, en el año 2020 necesitaremos más de 5 millones de años para poder visualizar el tráfico de video que circulará en un mes por las redes IP. En ese mismo año, más del $82 \%$ de todo el tráfico consumido en Internet será tráfico de video sobre IP. Además, los usuarios finales cada vez demandan mayor calidad, tanto a nivel de mayores resoluciones y se estima que para el 2020 más del $20 \%$ de video bajo demanda sea UHD (Ultra High Definition), como en el proceso de difusión del video a través de las redes IP.

Para poder satisfacer estos requerimientos de los usuarios finales en el campo de la difusión de contenido multimedia sobre redes IP, se tienen que llevar a cabo diversas tareas en diveros campos [2]. El primero de ellos es la resolución de las imágenes. Cada vez más se disponen de dispositivos con pantallas con mayor resolución y por tanto el usuario quiere disponer del contenido adecuado a esa misma resolución. Actualmente ya existen dispositivos con resoluciones de 8K UHDTV (Ultra High Definition TeleVision, 4320p), lo cual conlleva a crear contenido con estas resoluciones.

Ligado a esta característica, están los codecs de video y sus contenedores. Los codecs de video más utilizados para resoluciones inferiores a HD (High Definition, 720p) o FHD (Full High Definition, 1080p) son VP8 y $\mathrm{H} 264$, en cambio cuando las resoluciones aumentan los codecs que mejor se comportan son VP9 y H265 [3]. El último códec que pretende ser un éxito debido a su eficiencia y a su característica de software libre será AV1, ya que es la evolución de VP9 y existen grandes empresas, como son Cisco, Google, Intel, Microsoft, Netflix, que están apoyando el proyecto [4]. Unidos a los codecs están los contenedores y para la familia MPEG (H264 y H265) se utiliza el contenedor mp4, mientras que para la familia WebM (VP8 y VP9) se utiliza el contenedor webm.

Una vez preparado el video lo que se realiza es seleccionar los protocolos de transporte para la difusión del contenido multimedia. A día de hoy existen diversos protocolos para la difusión de contenido multimedia, pero en este articulo vamos a focalizarnos en los protocolos sobre HTTP, como son: HTTP Live Streaming (HLS), Adobe HTTP Dynamic Streaming (HDS), Microsoft Smooth Streaming (MSS) y Dynamic Adaptative Streaming over HTTP (DASH) [5]. Concretamente seleccionaremos DASH ya que es el protocolo estandarizado que mayor compatibilidad posee y además se trata del protocolo utilizado por plataformas como YouTube, Netflix, Amazon, Hulu, etc. 
Dado que el uso del protocolo DASH esta aumentando en los últimos años y es uno de los protocolos más utilizados para realizar streaming sobre HTTP. En este articulo presentamos la herramienta MediaDASH Tool, la cual es una aplicación web que permite de manera muy intuitiva poder comprimir y preparar videos para su difusión con DASH, así como su posterior visualización a través de la misma plataforma. Para ello abordaremos los siguientes objetivos:

Desarrollar una aplicación web que permita comprimir videos especificando diferentes características finales que el usuario desee que tenga el video, como resolución, tamaño, tasa binaria, etc.

- Preparar y reproducir videos en WebM, MP4, MPEG-DASH [6] y WebM-DASH [7], a través de la aplicación web.

- Llevar a cabo una evaluación de la herramienta para analizar el comportamiento de la misma haciento uso de los diversos métodos de video streaming.

Este artículo esta organizado de la siguiente manera. En la sección II trataremos el estado del arte. En primer lugar, hablaremos de los protocolos de streaming adaptativo sobre http más utilizados a día de hoy y posteriormente mostraremos plataformas para generar y difundir este tipo de contenido multimedia. En la sección III mostraremos el diseño de la aplicación MediaDASH Tool, sus diagramas de sequencia y la implementación. La implementación de la herramienta presentada en este artículo se mostrará en la sección IV y por último en la sección V se presentarán las conclusiones y los trabajos futuros que están naciendo a partir de esta aplicación.

\section{ESTADO DEL ARTE}

En este punto vamos a analizar dos aspectos claves para este articulo. En primer lugar, analizaremos las técnicas más utilizadas para la difusión adaptativa de video sobre HTTP y en segundo lugar comentaremos diversos trabajos que existen donde se presentan otras herramientas para la difusión de este tipo de contenidos, analizando cuales los son los puntos fuertes y débiles respecto a nuestra propuesta.

\section{A. Protocolos de streaming adaptativo sobre HTTP}

Actualmente, existen diversos protocolos para el streaming adaptativo que ofrecen diversas mejoras sobre los protocolos de transmisión tradicionales, como RTP o RTMP, entre las que se cuentan:

- Reducción de los costes de infraestructura.

- Posibilidad de almacenamiento en memoria caché en redes CDN y otras infraestructuras de caché HTTP.

- Reducción de las amenazas procedentes de restricciones de proxy y cortafuegos.

- Optimización en tiempo real mediante heurística en el equipo cliente (tasa de bits adaptativa).

- Redundancia integrada.
- Implementación sencilla de reproductores HTML5.

HLS [8] utiliza video H.264 MPEG-2 TS segmentado y archivos descriptores M3U8 para difundir el video en directo y a la carta, con tasas de bits adaptativas. Un archivo M3U8 es un índice que permite al cliente saber qué secuencias y segmentos están disponibles en un momento dado. El dispositivo selecciona automáticamente la secuencia más adecuada desde el archivo de manifiesto primario, teniendo en cuenta las limitaciones de ancho de banda y de CPU. A continuación, descarga el segmento y lo añade al búfer de reproducción. Otra alternativa similar propuesta por Adobe es HDS [9]. Este es el protocolo para la difusión de video adaptativo compatible con Flash. Este método de streaming permite envío de video adaptativo bajo demanda y en directo, con la principal diferencia con respecto a HLS que utiliza el contenedor MP4. Por otro lado, esta MSS [10] que es un método de entrega de medios híbridos desarrollado por Microsoft. Actúa como streaming, pero se basa en la descarga progresiva en HTTP. Las descargas HTTP se realizan en una serie de pequeños fragmentos, permitiendo que los medios se almacenen en caché de forma fácil y barata a lo largo de los puntos finales de la red, más cerca de los clientes. Proporcionar varias velocidades de bits codificadas de la misma fuente de medios, también permite a los clientes cambiar de forma continua y dinámica entre las velocidades de bits dependiendo de las condiciones de la red y de la potencia de la CPU.

Finalmente, DASH es un estándar ISO [6] lanzado con la intención de unificar la metodología de transmisión adaptativa. Hasta entonces cada plataforma, Micorsoft, Apple y Adobe, generaba cada uno los segmentos y los archivos de descripción con diferentes formatos, con lo que los dispositivos y programas que quisieran reproducirlos debían implementarlos todos. Para DASH, los archivos de audio y video se llaman MP (Media Presentation) y los archivos de descripción se llaman MPD (Media Presentation Description) y están codificados en XML. Al igual que las anteriores tecnologías de transmisión adaptativa mencionadas, estas consisten en codificar y trocear el contenido en diferentes bitrate para que el cliente solicite el más conveniente en cada momento. El archivo de descripción indexa este contenido. Su funcionamiento es el siguiente. Primero el cliente DASH solicita el archivo MPD, y con él obtiene toda la información necesaria de los videos. A continuación, a través de peticiones HTTP el cliente captura los segmentos de los videos y a la vez monitoriza la red obteniendo así el stream más adecuado al estado de la red.

\section{B. Plataformas de video streaming adaptativo}

En el año 2015 YouTube decidió dejar de lado el reproductor web basado en Flash y pasar a un reproductor basado en HTML5. Desde ese momento también hubo un cambio en la forma de transmitir el video a través de HTTP, y se seleccionó DASH para realizar el streaming adaptativo del contenido que ofrecía YouTube 
[11]. YouTube es una plataforma web cuyo objetivo es realizar la codificación, compresión y recepción de los videos que incluyen los usuarios. Estos aspectos son muy similares a la finalidad de la aplicación propuesta, pero la principal diferencia con el sistema propuesto es que el usuario no puedo modificar la codificación de los streams. En cambio, en la herramienta MediaDASH Tool desarrollada, el usuario tiene un control total sobre su contenido. Otra plataforma de video bajo demanda que está utilizando DASH es Netflix [12]. En este caso la plataforma web de Netflix sólo permite reproducir sus videos y los usuarios finales no pueden incluir su propio contenido. La última plataforma en utilizar DASH ha sido Hulu [13]. Esta plataforma era un poco reticente a cambiar su forma de realizar streaming basado en FLV sobre RTMP o HLS para dispositivos Apple, pero finalmente ha decidio cambiar a DASH para así tener una mayor compatibilidad. En esta plataforma ocurre lo mismo que con Netflix, donde el usuario es un simple consumidor de contenido multimedia.

Finalmente, hemos encontrado un trabajo [14] donde los autores presentan una plataforma para realizar streaming DASH. Esta aplicación permite la configuración de los pasos necesarios a lo largo de la comunicación multimedia extremo a extremo, desde la codificación, segmentación y almacenamiento del contenido multimedia en el lado del servidor, hasta la entrega y consumo adaptativo del streaming multimedia en el lado del cliente. Esta aplicación esta desarrollada mediante el framework Gstreamer y su programación ha sido en C. Las principales diferencias entre esta aplicación y la presentada en este articulo son: MediaDASH Tool es una herramienta web (uso masivo independiente del dispositivo y el sistema operativo) y las funcionalidades de servidor/cliente está en la misma aplicación, lo que aporta un uso más fácil e intuitivo de la aplicación por usuarios sin conocimientos sobre streaming. Nuestra herramienta puedo trabajar con codecs VP8, VP9, H64 y H265, en cambio el trabajo [14] solo utiliza H264. Por otra parte, el trabajo [14] puede emular condiciones de red para observar cambios en el streaming, aspecto que en nuestra aplicación no se ha incluido en la propia aplicación.

\section{MEDIADASH TOOL}

MediaDASH Tool es una aplicación web responsiva a la que se puede acceder desde cualquier PC o dispositivo móvil (Smartphone o Tablet) conectado a la red a través del protocolo de aplicación HTTP. Al acceder a la aplicación, primero se accederá a una página inicial donde el usuario se podrá registrar, iniciar sesión o ver los videos públicos disponibles. Cuando un usuario registrado inicia la sesión se pasa a la página inicial de usuario registrado donde se puede acceder a las distintas acciones que se pueden realizar, ya sea codificar o administrar videos o archivos. Las acciones que se pueden ejecutar son las siguientes:

- Cargar un video o archivo desde el dispositivo que accede a la aplicación.

- Ver la lista de archivos o videos de los que se es propietario y también de los que son públicos.

- Para la codificación se accede a un pequeño formulario donde se pueden especificar algunas de las características que se desea que tenga el video final. Se puede especificar el bitrate, la resolución, la calidad en la compresión, el tamaño de GOP, el estándar de codificación a utilizar y el formato de video de salida.

- Para la codificación en DASH también se puede especificar cuantos streams se quieren crear, y la resolución cada uno de ellos. Este proceso además de crear dichos videos creará automáticamente el archivo MPD necesario para acceder a los videos DASH.

- $\quad$ Se pueden reproducir archivos codificados en H.264, WebM, MPEG-DASH y WebM-DASH.

Existen una serie de restricciones para las acciones mencionadas anterioremente:

- La resolución de salida no podrá ser superior a la del archivo original.

- Se mantendrá la relación de aspecto (DAR) si la original se encuentra entre las disponibles, y en el caso que no exista en nuestra base de datos se utilizará la de 16:9 por defecto.

- Las características que se seleccionen se combinarán siempre que se pueda, para que así la codificación pueda llevarse a cabo de forma correcta. Si en una codificación, alguna de las características no es compatible, no se realizará y se le notificará al usuario.

- Los estándares de codificación, la resolución y el formato de video de salida se escogerán de las opciones que se indroducen en el formulario, pudiendo elegir una opción no especificada lo que provocará que algunas de las opciones se elijan automáticamente.

La parte del backend de la aplicación web está programada en PHP y JavaScript. Para las pruebas hemos utilizado un servidor Apache/MySQL. Para la codificación se necesitan instalar en el sistema operativo (SO) del servidor las herramientas FFmpeg [15] y MP4Box [16], las cuales se lanzarán desde la web por línea de comandos de forma transparente para el usuario, el cual especificará las características de la codificación a través de un formulario web.

\section{A. Casos de uso}

A raíz de la especificación del sistema definidos anteriormente, podemos obtener los siguientes perfiles que usarán el sistema y los casos de uso que habrá. los usuarios del sistema se han separado en tres perfiles:

Administrador: es un usuario que podrá acceder a la totalidad de los videos y a la información de los usuarios y podrá borrar videos o usuarios si lo cree conveniente. 
- Usuario no registrado: es el tipo de usuario con el que se accede a la página de inicio. Éste usuario podrá registrarse o hacer login. Es un usuario de visita de la página, que podrá ver y reproducir los videos que sean públicos.

- Usuario registrado: es el usuario que se registra para hacer uso de las funcionalidades de codificación de la página.

Debemos de diferenciar entre videos y archivos, siendo los videos archivos multimedia reproducibles, y los archivos corresponden a archivos (valga la redundancia) con extensión y $4 \mathrm{~m}$, que son un conjunto de frames en crudo con formato video YCbCr. Los archivos una vez codificados pasan a crear un nuevo video manteniendo el archivo y4m original. En la Fig. 1 se observa la relación entre los perfiles de los usuarios y la relación de los casos de usos definidos en la aplicación.

\section{B. Diseño de la estructura web}

Para poder implementar la aplicación web vamos a presentar el diseño de la estructura de la web y el acceso a las diferentes funcionalidades basado en niveles. Como se puede observar en la Fig. 2, la estructura esta dividida en 3 niveles. En el Nivel 0, la página inicial que se verá cuando accedes a la web será la de inicio. Desde esa página, mostraremos los videos públicos y podremos acceder a la página de registro y de login. Estas dos páginas también pertenecerán al nivel 0 , dado que son páginas que utilizaremos de paso al siguiente nivel.

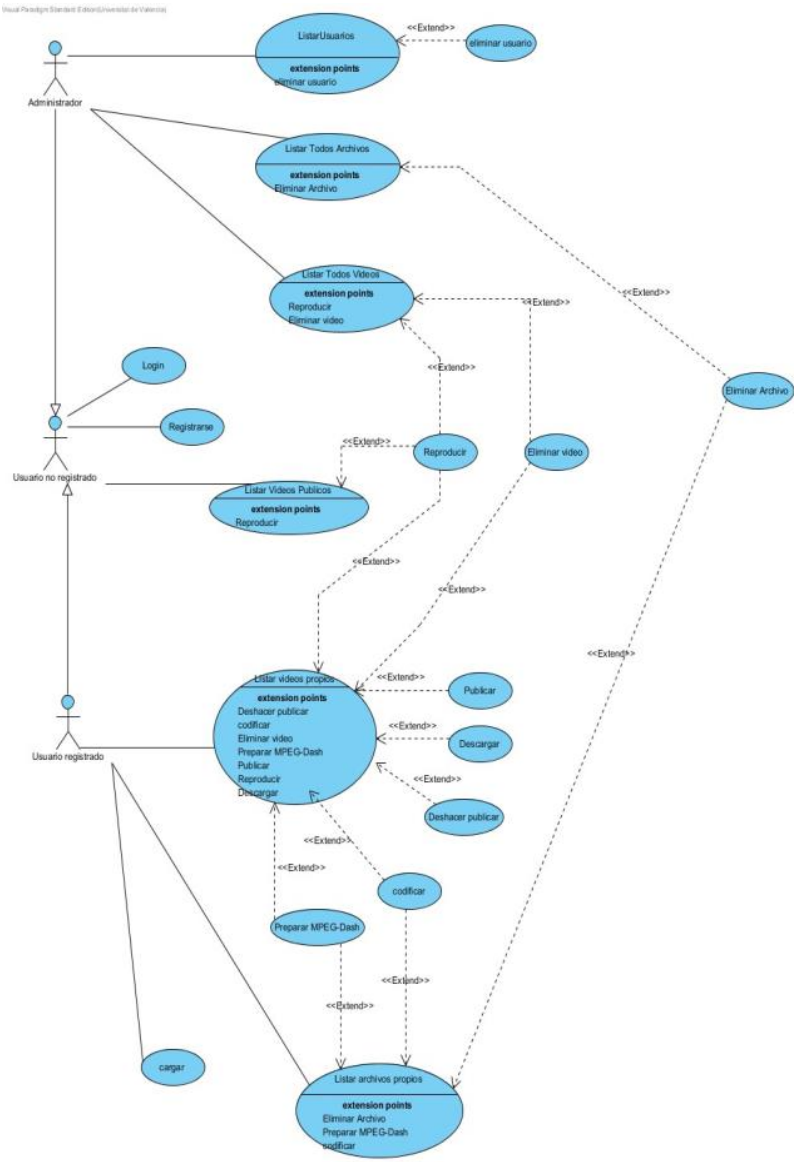

Fig. 1. Esquema de casos de uso de la aplicación MediaDASH Tool.

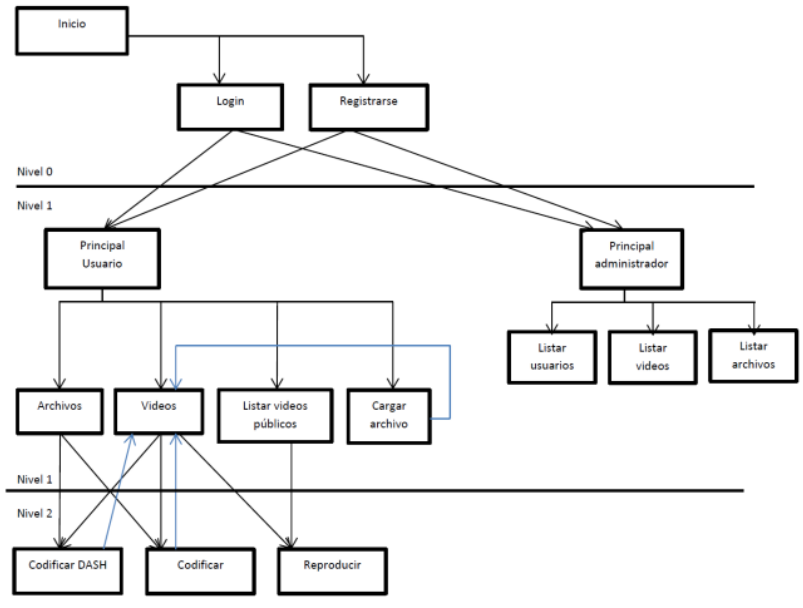

Fig. 2. Estructura de la web.

Desde el nivel 0 podremos acceder al nivel 1. Este nivel se separa en 2 partes, según el tipo de usuario con el que se haya realizado el login, administrador o usuario. En ambas partes se accederá a una página principal de presentación y de menú, desde la cual únicamente se podrá acceder a las páginas de este mismo nivel o cerrar la sesión. Si se trata de un usuario registrado, podrás acceder a las páginas de listar videos, listar archivos, cargar archivo y listar videos públicos. Por otro lado, si eres administrador podrás acceder a las páginas de listar videos, listar archivos y listar usuarios.

Por último, estarán las páginas del nivel 2. A estas páginas se podrá acceder sólo desde el nivel 1. Serán las páginas desde las cuales se lanzarán las codificaciones o las reproducciones.

\section{Diseño de la estructura de datos}

La estructura de los datos consta de 7 tablas implementadas en MySQL con la siguiente información. La tabla usuarios almacena la información de cada uno de los usuarios, como su usuario, que es con él que se realiza el login y una contraseña. También se guarda un correo electrónico que se utilizará como método de contacto en el caso que el administrador borre su cuenta o alguno de sus archivos. Para diferenciar si el usuario es administrador o no, habrá un último campo que será el de rol. Las funciones que se pueden realizar sobre esta tabla son añadir usuario, eliminar usuario, y consultar datos para hacer el login.

En la tabla archivos subidos se almacena la información necesaria para el manejo de los archivos y4m. En ella, guardaremos el nombre, el usuario al que pertenece, la URL relativa que hace referencia a la ubicación del archivo para poder trabajar con él y por último el formato. En ellas, se puede añadir, borrar, codificar archivos, descargar y ver la información. La codificación, la descarga y la consulta de la información no es sobre los datos, sino sobre el archivo al que hacen referencia.

En la tabla videos se almacena lo mismo que para archivos subidos, es decir, nombre, usuario, formato y URL, añadiéndole la característica de si es público o no. Esta última característica no se encuentra en la tabla 
archivos porque estos archivos sólo pueden ser usados por sus propietarios. También se guarda el nombre de una imagen, que será el de una captura que realicemos sobre el video para poder realizar una previsualización del mismo. Sobre los videos podremos añadir y eliminar, cambiar el estado del atributo "público", reproducir, descargar, codificar y consultar información. Al igual que en archivos, la reproducción, la descarga, la codificación y la consulta de información se realizan sobre el archivo de video al que hacen referencia.

La tabla MPD guarda el archivo mdp que contiene la misma información que la tabla videos. Esto es porque el fichero mpd se utiliza para reproducir videos DASH, y es el archivo que se carga cuando elegimos la opción de reproducir, así que, aunque no sean archivos de video, los tratamos como tal. Por lo que guardamos el nombre, el usuario, si es público, una captura, la URL y el formato, si es WebM o MP4. Sobre la tabla MPD se podrá añadir, eliminar, reproducir, publicar y ver la información.

La tabla Videos Dash guarda información sobre los videos que se han codificado para transmitir en DASH. Se guardará el nombre, el usuario, el formato, la URL, si es público, el MPD que le hace referencia. A estos videos el usuario no podrá acceder directamente, sino solo a través de su MPD. Se puede añadir, borrar, reproducir y ver información.

La relación entre estas tablas la podemos ver en la Fig. 3. La estructura de datos utilizada es una estructura simple. La relación que hay entre ellas es que cada video, archivo, MPD y video Dash pertenece a un usuario. En la implementación de la base de datos, el atributo usuario que tiene cada uno de estas tablas será la clave ajena a la tabla usuarios, aplicando así la restricción de que cada archivo pertenece a un usuario obligatoriamente. También existe la relación entre la tabla $M P D$ y la tabla Videos DASH. Esta relación es para que cada video DASH sea utilizado solamente por un mpd. Cada mpd puede referenciar a uno o más videos DASH.

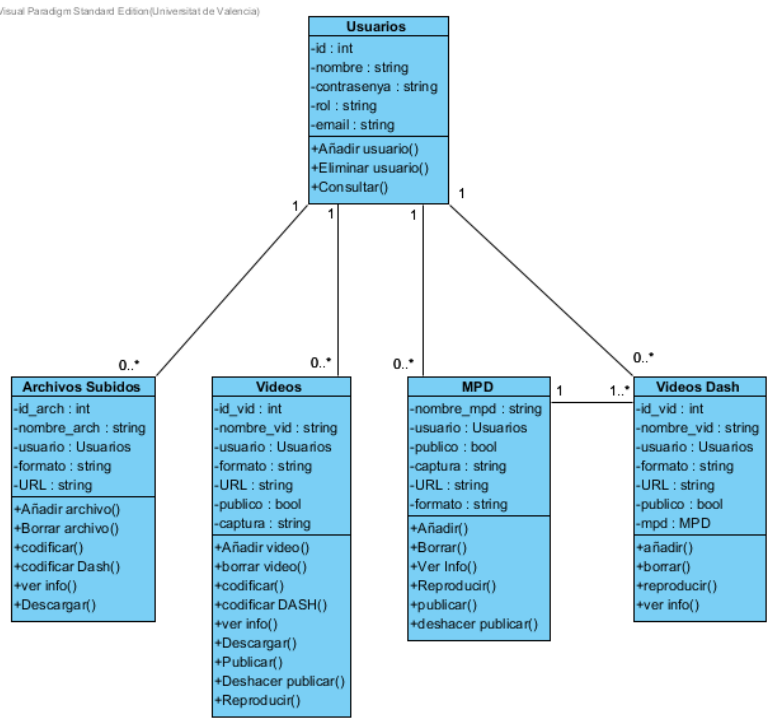

Fig. 3. Base de datos relacional utilizada en MediaDASH Tool.
A parte de estas tablas, hay dos tablas que no estarán relacionadas con ninguna. Son la tabla de resoluciones y de nombres reservados. La tabla resoluciones guarda una lista no modificable de resoluciones disponibles y sus respectivos DAR. La utilizaremos para proveer de una lista de resoluciones entre las que se podrá elegir para llevar a cabo una codificación. Cuando queramos hacer una codificación, buscaremos el DAR del archivo origen y buscaremos en la tabla qué resoluciones hay disponibles con ese DAR. Así podremos cambiar la resolución manteniendo el DAR original. Sobre esta tabla, sólo se podrán realizar consultas. La tabla nombres reservados, como su nombre indica, es una tabla donde se guardará temporalmente los nombres de archivos mientras se realiza una carga o codificación, para evitar posteriormente conflictos entre archivos con el mismo nombre.

\section{Diagramas de Sequencia}

Para entender cómo se implementan las codificaciones, vamos a mostrar los pasos que se siguen para llevarlas a cabo, y vamos a plasmarlos en unos diagramas de secuencia (ver Fig. 4 y Fig. 5).

En la Fig. 4 vemos una codificación de un video que no sea DASH. Para empezar el usuario, desde la lista de videos, selecciona la opción de codificar (suponemos un archivo o video ya subido al servidor). Al solicitar codificar, la aplicación envía al usuario registrado al formulario correspondiente para que rellene los campos con las características que desea que tenga el video. Cuando el usuario rellena los campos y lanza la codificación primero, se comprueba que el nombre que le quiere poner al nuevo video no existe. Si existe se le solicita que introduzca uno nuevo nombre. Si no existe, entonces la aplicación lanza FFmpeg pasándole los datos del formulario y el nombre del video de origen y éste empieza la codificación. Cuando acaba la codificación FFmpeg habrá creado un nuevo video. Este se añade a la lista de videos. Cuando se confirma la codificación, la aplicación lista los videos al usuario, incluyendo el nuevo video en la lista

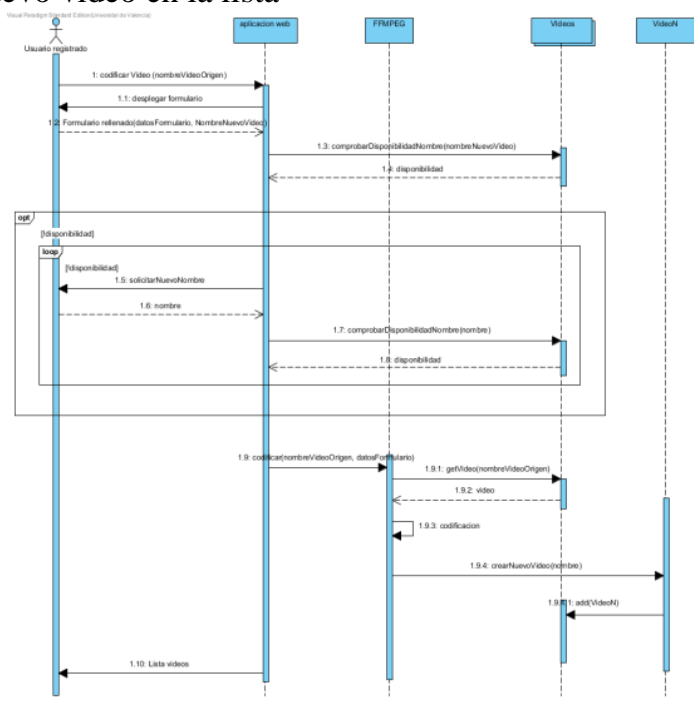

Fig. 4. Diagrama de sequencia de la codificación de un video. 


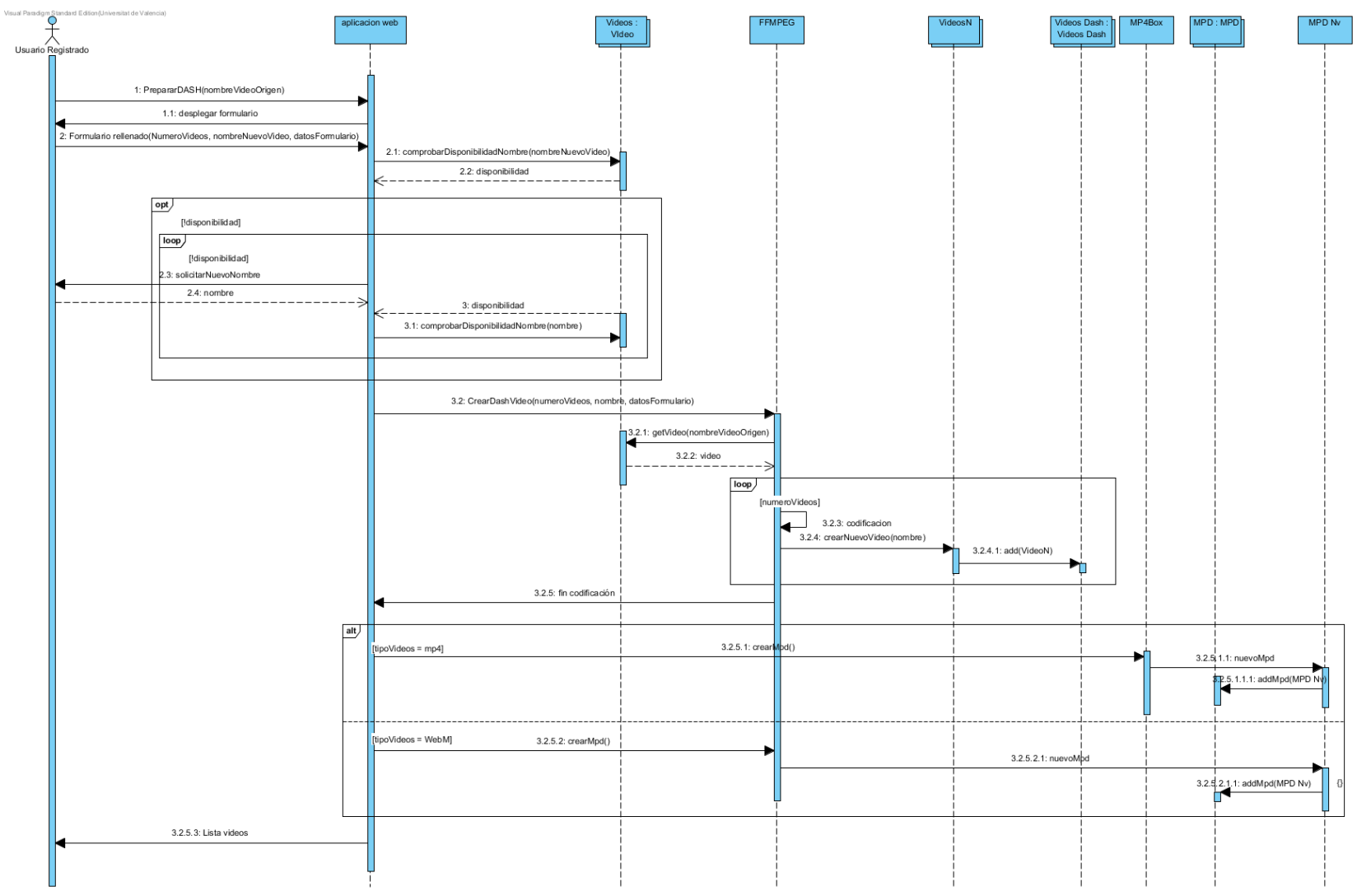

Fig. 5. Diagrama de sequencia de la codificación de un video DASH.

Cuando codificamos en DASH, se codifican varios videos y se generan los archivos MPD (ver Fig. 5). Después de que el usuario introduce los datos en el formulario y de comprobar que el nombre que desea ponerle está disponible, empieza la codificación y FFmpeg codifica varios videos. Después cuando FFmpeg confirma que ha terminado la codificació de todos los videos, entonces la aplicación lanza MP4Box para generar el archivo MPD si los videos son en formato mp4 o lanza otra vez FFmpeg si los videos son en formato WebM, para que genere el MPD correspondiente al DASH generado. Después los videos nuevos creados se añaden a la lista de videos Dash, y el MPD a la lista de MPDs.

\section{IV.IMPLEMENTACIÓN DE MEDIADASH TOOL}

La implementación web de la herramienta MediaDASH Tool se ha realizado utilizando HTML5, JavaScript, PHP y MySQL, mientras que la parte correspondiente a la codificación se ha utilizado el framework FFmpeg y MP4Box. Parte del fronted de la aplicación web MediaDASH Tool puede observarse en las Fig. 6 - 15. En la Fig. 6 puede verse la vista de la aplicación de un usuario no registrado, que podrá seleccionar el video público a reproducir y reproducirlo (veasé Fig. 7).

En la Fig. 8 vemos la aplicación tal y como la manejaría un usuario registrado. En ella se puede ver un menú a la izquierda donde se puede seleccionar los videos públicos, los videos precodificados, los videos en crudo (raw) y la acción de subir un video. Todos los videos disponen de una imagen previa para tener una visión previa del video excepto los videos o archivos que estén en fase de codificación, donde en lugar de la imagen del video aparecerá una imagen indicando que se están codificando o lo que no poseen imagen previa que se indicará que la imagen no está disponible. Además en la Fig. 9 se observan las acciones que se pueden realizar sobre un archivo (video raw) o un video, que son la codificación normal, codificación DASH, despublicar o publicar, descargar y obtener información del archivo.

En la Fig. 10 observamos el formulario para la codificación DASH de un video. En este formulario se puede seleccionar el códec, los frames por segundo, el tamaño de GOP, y para cada stream la resolución y el bitrate, además de incluir un nombre para el archivo MPD que será el que permite el streming del video DASH. En la Fig. 11 se puede observar el reproductor que dispone el usuario registrado.

En la Fig. 12 vemos el frontend del usuario administrador, en el aparecen 3 nuevos menús que son usuarios, todos los videos y todos los archivos. En el menú usuarios (ver Fig. 13) aparece la gestión de usuarios registrados en la herramienta. En el resto de menus nuevos se visualizan todos los videos o archivos y se pueden eliminar o mostrar la información (ver Fig. 14). Por último, en la Fig. 15 se observa el proceso de subido de un fichero, el cual puede realizar el usuario regisrado y el administrador. 


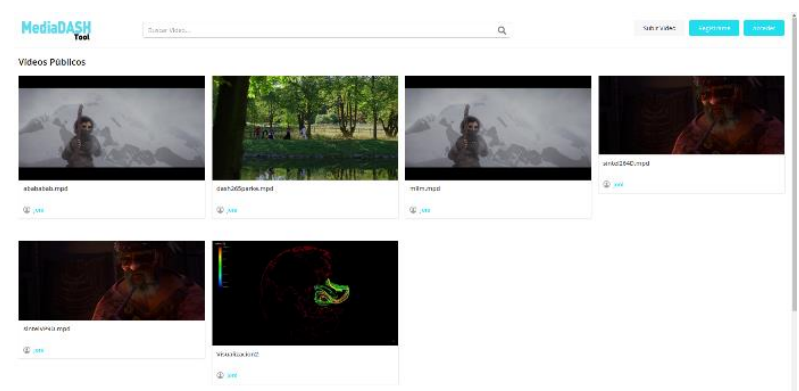

Fig. 6. Frontend con usuario público.

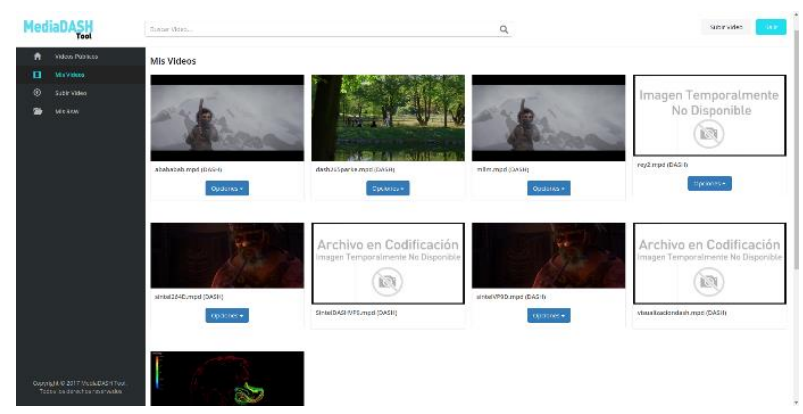

Fig. 8. Videos propiedad de un usuario registrado.

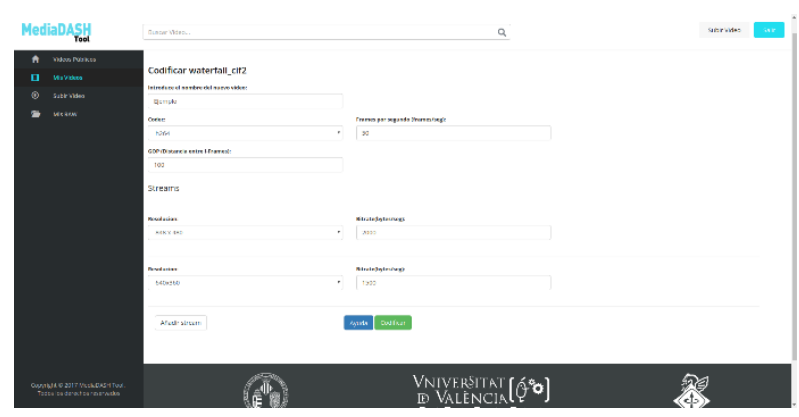

Fig. 10. Formulario para la codificación DASH.

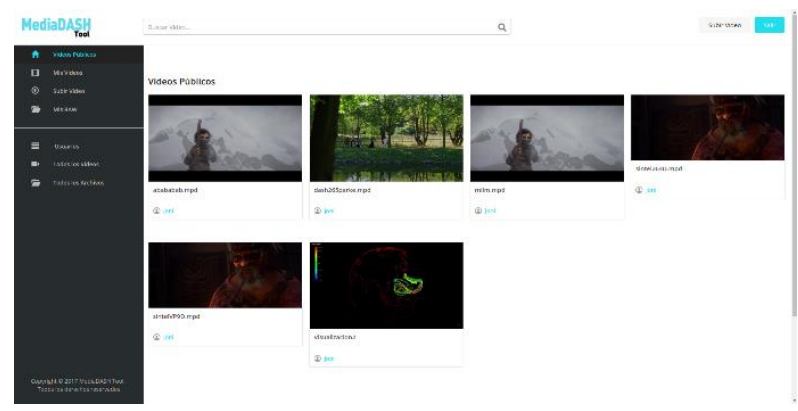

Fig. 12. Frontend para el usuario administrador.

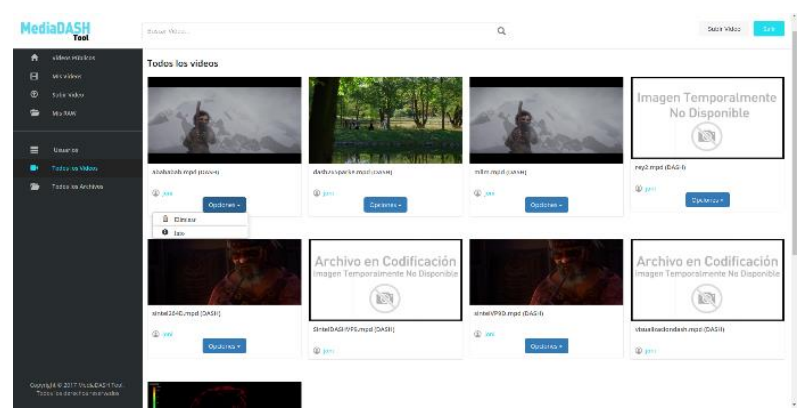

Fig. 14. Acciones que pueden realizar el usuario administrador.

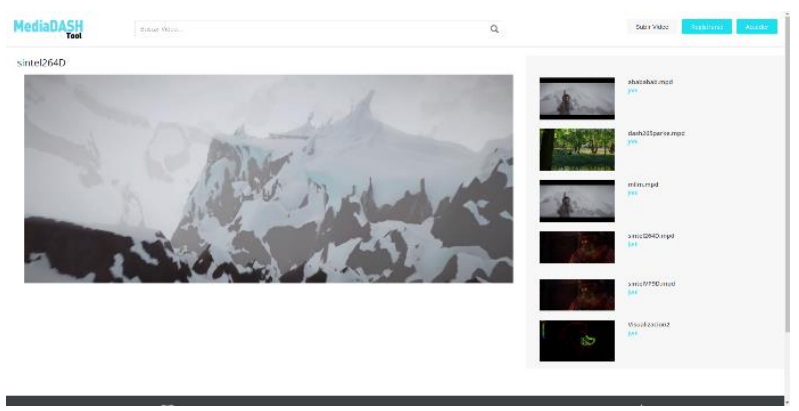

Fig. 7. Visualización de video DASH con usuario público.

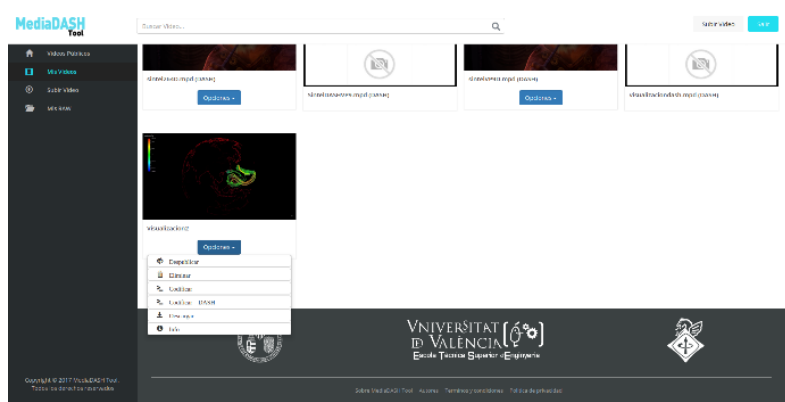

Fig. 9. Acciones aplicables a los videos de un usuario registrado.

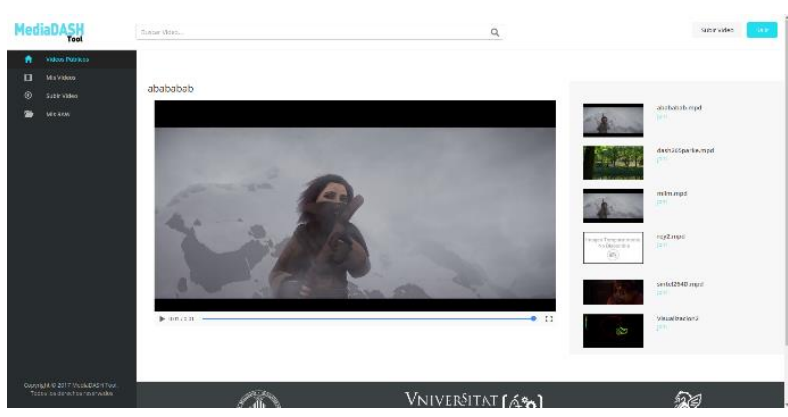

Fig. 11. Visualización de video DASH de un usuario registrado.

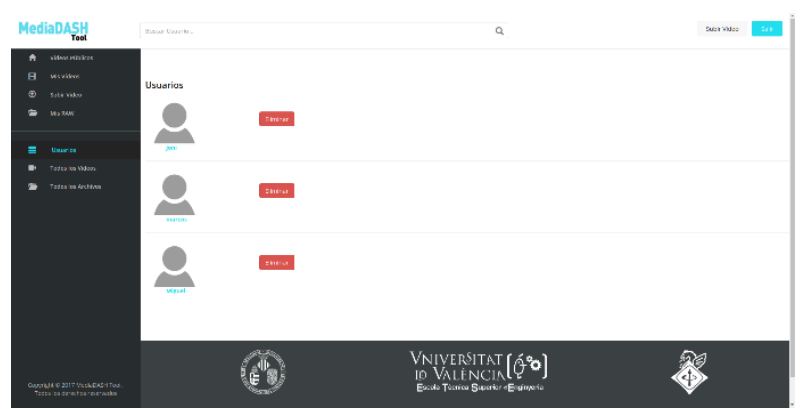

Fig. 13. Gestión de usuarios para el usuario administrador.

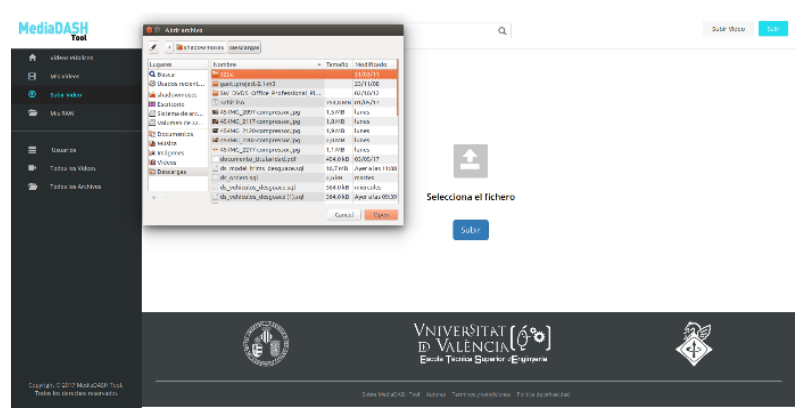

Fig. 15. Proceso de subida de archivos o videos al servidor. 


\section{CONCluSiONES Y TRABAJO FUTURO}

En este artículo hemos presentada la herramienta MediaDASH Tool, la cual es una aplicación web que permite de manera muy intuitiva poder comprimir y preparar videos para su difusión con DASH. Así como su posterior visualización a través de la misma plataforma. Esta herramienta puede ser de gran utilidad para usuarios finales que quieran disponer de su sistema DASH así como para investigadores que quieran testear sus contenidos multimedia haciendo uso de estas técnicas y servicios.

MediaDASH Tool es una herramienta web responsiva (uso masivo independiente del dispositivo y el sistema operativo) y las funcionalidades de servidor/cliente esta en la misma aplicación lo que aporta un uso más fácil e intuitivo de la aplicación por usuarios sin conocimientos sobre streaming. Nuestra herramienta puedo trabajar con codecs como VP8, VP9 encapsulados en WebM y H264/AVC, H265/HEVC encapsulados en MP4.

Como trabajo futuro estamos mejorando la aplicación para que disponga de más características como puede ser la monitorización del video recibido por el usuario final y el almacenamiento de esta información para su posible estudio posterior.

\section{AGRADECIMIENTOS}

Este trabajo ha sido parcialmente subvencionado por la Universitat de València a través de los proyectos UVINV-PRECOMP14-207134, UV-INVAE15-339582, por la Generalitat Valenciana a través del proyecto GV2016-002 y por el Ministerio de Economía a través del proyecto BIA2016-76957-C3-1-R.

\section{REFERENCIAS}

[1] Cisco Systems, «Cisco Visual Networking Index: Forecast and Methodology, 2016-2021,» 7 Junio 2017. [En línea]. Available: http://www.cisco.com/c/en/us/solutions/collateral/serviceprovider/visual-networking-index-vni/complete-white-paperc11-481360.html. [Último acceso: Mayo 2017].

[2] B. Bing, Next-generation video coding and streaming, New Jersey: John Wiley \& Sons, 2015.

[3] J. Bienik, M. Uhrina, M. Kuba y M. Vaculik, «Performance of H. 264, H. 265, VP8 and VP9 Compression Standards for High Resolutions,» de 19th International Conference on Network-
Based Information Systems (NBiS), Ostrava, Czech Republic, 2016.

[4] Alliance for Open Media, «Alliance for Open Media,» 2015. [En línea]. Available: http://aomedia.org. [Último acceso: Mayo 2017].

[5] M. Seufert, S. Egger, M. Slanina, T. Zinner, T. Hobfeld y P. TranGia, «A survey on quality of experience of HTTP adaptive streaming,» IEEE Communications Surveys \& Tutorials, vol. 17, no 1, pp. 469-492, 2015.

[6] ISO, «ISO/IEC 23009-1:2014. Information technology -- Dynamic adaptive streaming over HTTP (DASH) -- Part 1: Media presentation description and segment formats,» 2014.

[7] WebM Project, «WebM Dash Specification,» [En línea]. Available: http://wiki.webmproject.org/adaptivestreaming/webm-dash-specification. [Último acceso: 25 Julio 2017].

[8] Apple Inc., «HTTP Live Streaming,» 2016. [En línea]. Available: https://developer.apple.com/library/content/documentation/Net workingInternet/Conceptual/StreamingMediaGuide/Introductio n/Introduction.html. [Último acceso: 105 2017].

[9] Adobe, «HTTP Dynamic Streaming Specification,» 2013. [En línea]. Available: $\mathrm{http}: / /$ wwwimages.adobe.com/content/dam/Adobe/en/devnet/h ds/pdfs/adobe-hds-specification.pdf. [Último acceso: 10 Mayo 2017].

[10] Microsoft, «Smooth streaming,» 2008. [En línea]. Available: https://www.iis.net/learn/media/on-demand-smoothstreaming/smooth-streaming-technical-overview . [Último acceso: 10 Mayo 2017].

[11] D. K. Krishnappa, D. Bhat y M. Zink, «DASHing YouTube: An analysis of using DASH in YouTube video service,» de In IEEE 38th Conference on Local Computer Networks (LCN), Sydney, Australia, 2013.

[12] J. Martin, Y. Fu, N. Wourms y T. Shaw, «Characterizing Netflix bandwidth consumption,» de $n 2013$ IEEE Consumer Communications and Networking Conference (CCNC), Las Vegas, USA, 2013.

[13] N. Weil , «Hulu’s Move to DASH,» 2015. [En línea]. Available: http://www.streamingmediaglobal.com/Articles/ReadArticle.as px?ArticleID=105110\&PageNum=1. [Último acceso: 10 MAyo 2017].

[14] D. Gómez, F. Boronat, M. Montagud y C. Luzón, «End-to-end DASH platform including a network-based and client-based adaptive quality switching module,» de In Proceedings of the 7th International Conference on Multimedia Systems, Klagenfurt am Wörthersee, Austria, 2016.

[15] FFmpeg, «FFmpeg multimedia framework,» [En línea]. Available: https://ffmpeg.org. [Último acceso: 10 Mayo 2017].

[16] GPAC, «MP4Box multimedia packager,» [En línea]. Available: https://gpac.wp.imt.fr/mp4box/. [Último acceso: 10 Mayo 2017]. 